\title{
Activity Monitoring in Heart Failure Patients With Cardiac Resynchronization Therapy
}

\author{
Mihoko Kawabata, MD, PhD *; ; Cecilia Fantoni, MD*,**; Francois Regoli, MD*; \\ Santi Raffa, MD*; Francesca Pastori, MD*; Simona Fratini, MD, PhD*; \\ John Prentice, $\mathrm{PhD}^{\dagger}$; Helmut U. Klein, $\mathrm{MD}^{*}$; Angelo Auricchio, $\mathrm{MD}, \mathrm{PhD}^{*}, \dagger$
}

\begin{abstract}
Background Cardiac resynchronization therapy (CRT) improves functional capacity in heart failure patients. This study aimed to prospectively analyze long-term device-based monitoring of physical activity in patients undergoing CRT.

Methods and Results The Activity Log Index (ALI), calculated by CRT devices, represents the percentage of time when acceleration exceeds a threshold and monitors the physical activity. Data from 178 CRT patients (New York Heart Association III 91\%, left ventricular ejection fraction $21 \pm 6 \%$, left ventricular end-diastolic diameter $69 \pm 9 \mathrm{~mm}$, QRS $159 \pm 27 \mathrm{~ms}$, sinus rhythm $81 \%$ ) were retrieved. The ALI increased from a baseline value of $3.6 \pm$ 2.0 to $11.2 \pm 4.6(\mathrm{p}<0.005) 104$ weeks after initiation of CRT. A plateau was reached at approximately 12 weeks and thereafter ALI remained stable for up to 2 years. The magnitude of the changes in ALI was similar in patients with different etiologies and underlying rhythms. Despite similar values at baseline, elderly patients ( $\geq 65$ years) exhibited significantly lower ALI values than younger patients during the follow-up and at the plateau $(9.5 \pm 4.2$ vs $13.3 \pm 4.8, \mathrm{p}<0.001)$.
\end{abstract}

Conclusions Device-based monitoring of physical activity in CRT patients is feasible. CRT resulted in a large and long-term increase in physical activity. (Circ J 2007; 71: 1885-1892)

Key Words: Automatic monitoring; Cardiac resynchronization therapy; Heart failure; Physical activity; Ventricular conduction delay

$\mathbf{E}$ xercise intolerance is a hallmark of heart failure (HF) and is predictive of all-cause mortality and hospitalizations ${ }^{1,2}$ Discouraging a sedentary lifestyle and promoting physical activity have both been shown to improve functional capacity, quality of life, and clinical outcome? 4

Cardiac resynchronization therapy (CRT) restores the cardiac electro-mechanical synchrony, leading to improved pumping function in HF patients with ventricular conduction disturbances. Consequently, CRT is able to increase functional capacity, exercise tolerance, 5,6 and aerobic capacity? to delay the onset of anaerobic metabolism5 and to decrease the sympathetic drive of the heart? All these effects have been reported mainly in CRT patients with sinus rhythm (SR) within a few months after device implantation, 5 Long-term exercise data in HF patients treated with CRT are limited. Furthermore, etiology-, rhythm-, diabetes-, and age-related differences in the improvement in function-

(Received November 2, 2006; revised manuscript received July 2, 2007; accepted August 30, 2007)

Department of Cardiovascular Medicine, Tokyo Medical and Dental University, Tokyo, Japan, *Division of Cardiology, University Hospital, Magdeburg, Germany, **Division of Cardiovascular Interventional Radiology, IRCCS Policlinico San Donato, San Donato Milanese, Italy, "Wild Rose Biophysics, Boulder, CO, USA and Department of Cardiology, Fondazione Cardiocentro Ticino, Lugano, Switzerland

This paper was awarded "The Ken-ichi Harumi Young Investigator" award at the $32^{\text {nd }}$ International Congress of Electrocardiology, Gdansk, June $4^{\text {th }}, 2005$, presented by Dr Fantoni.

Mailing address: Mihoko Kawabata, MD, PhD, Department of Cardiovascular Medicine, Tokyo Medical and Dental University, 1-5-45 Yushima, Bunkyo-ku, Tokyo 113-8519, Japan. E-mail: mihoko_ kawabata.cvm@tmd.ac.jp al capacity after CRT have not yet been fully evaluated.

The 6-min walking test is frequently used to assess submaximal exercise capacity in HF patients treated with CRT 5 ,9 However, the distance walked is influenced by several factors, including patient motivation, coaching, anatomical limitations, and physical training. Furthermore, in HF patients variability in the distance walked between 2 subsequent tests is frequently observed $!^{10}$ In contrast, symptom-limited exercise testing, eventually combined with an assessment of oxygen consumption, is influenced by familiarity with the test and may not reflect the patient's spontaneous daily physical activity, plus its routine determination is costly and time-consuming.

Recent technological advances have made device-based monitoring of physical activity possible using data collected through the device's sensors!1 Short-term, continuous, device-based monitoring of physical activity has proven to be feasible, although in a limited number of patients to date ${ }^{12}$ Because each device uses proprietary technology and analysis algorithms, the results obtained with 1 device may not be entirely applicable to other devices. Finally, whether this monitoring remains of value over the long term and in different subgroups of HF patients is unknown. It has been shown that the Activity Log Index (ALI), which quantifies body movement based on data derived from accelerometers built into the device, reliably tracks changes in the daily, spontaneous physical activity of patients with advanced HF. In addition, the ALI is well correlated with the distance walked within $6 \mathrm{~min} ! 1$

The aim of this study was to prospectively investigate the validity of long-term device-based monitoring of physical activity in advanced HF patients treated with CRT. Further- 
Table 1 Clinical Characteristics of the Study Population

\begin{tabular}{|c|c|c|c|c|}
\hline & All patients & $\begin{array}{l}\text { De-novo CRT } \\
\text { implantation }\end{array}$ & Device replacement & $p$ value \\
\hline No. of patients & 178 & 147 & 31 & \\
\hline Male $(\%)$ & 80 & 82 & 70 & $N S$ \\
\hline Age (years) & $65 \pm 8$ & $65 \pm 9$ & $63 \pm 6$ & NS \\
\hline Height $(m)$ & $1.72 \pm 0.09$ & $1.73 \pm 0.08$ & $1.68 \pm 0.1$ & $<0.05$ \\
\hline Weight (kg) & $80 \pm 13$ & $81 \pm 14$ & $73 \pm 10$ & $N S$ \\
\hline$C A D(\%)$ & 60 & 62 & 43 & $<0.05$ \\
\hline Diabetes (\%) & 41 & 39 & 50 & $N S$ \\
\hline NYHA functional class III/IV & $162 / 16$ & $132 / 15$ & $30 / 1$ & $N S$ \\
\hline Ejection fraction $(\%)$ & $21 \pm 6$ & $21 \pm 7$ & $22 \pm 6$ & $N S$ \\
\hline $\operatorname{LVEDD}(\mathrm{mm})$ & $69 \pm 9$ & $69 \pm 9$ & $69 \pm 9$ & NS \\
\hline Peak $\mathrm{VO}_{2}\left(\mathrm{ml} \cdot \mathrm{kg}^{-1} \cdot \mathrm{min}^{-1}\right)$ & $13.2 \pm 4.1$ & $13.4 \pm 4.2$ & $12.0 \pm 4.4$ & $N S$ \\
\hline Sinus rhythm (\%) & 81 & 82 & 77 & $N S$ \\
\hline QRS duration (ms) & $159 \pm 27$ & $158 \pm 27$ & $163 \pm 27$ & $N S$ \\
\hline Mean PCWP $(m m H g)$ & $16 \pm 10$ & $16 \pm 10$ & $16 \pm 10$ & NS \\
\hline Cardiac index $\left(L \cdot \mathrm{min}^{-1} \cdot \mathrm{m}^{-2}\right)$ & $2.2 \pm 0.6$ & $2.2 \pm 0.8$ & $2.3 \pm 0.2$ & $N S$ \\
\hline$A C E I / A R B(\%)$ & 98 & 98 & 100 & NS \\
\hline$\beta$-blockers (\%) & 96 & 96 & 97 & $N S$ \\
\hline Aldosterone-antagonists (\%) & 38 & 42 & 20 & $<0.05$ \\
\hline Digitalis (\%) & 55 & 51 & 70 & $<0.05$ \\
\hline Amiodarone (\%) & 16 & 15 & 20 & $N S$ \\
\hline$C R T-D(\%)$ & 83 & 90 & 53 & $<0.01$ \\
\hline
\end{tabular}

$P$ values refer to the comparison between de-novo CRT and re-implantation patients.

CRT, cardiac resynchronisation therapy; CAD, coronary artery disease; NYHA, New York Heart Association; LVEDD, left ventricular end-diastolic diameter; peak $\dot{V} \mathrm{O}_{2}$, oxygen consumption at peak exercise; PCWP, pulmonary capillary wedge pressure; ACEI, angiotensin-cobverting-enzyme inhibitor; ARB, angiotensin-receptor blockers; CRT-D, cardiac resynchronisation therapy device with defibrillator back-up.

more, we focused on etiology-, rhythm-, diabetes-, and agerelated differences in the improvement in physical activity after CRT.

\section{Methods}

\section{Patient Population}

Data from device-based monitoring of the physical activity of 178 consecutive patients with moderate to severe HF and a ventricular conduction delay (QRS duration $\geq 120 \mathrm{~ms}$ ) who were treated with CRT were prospectively collected. The indication for CRT implantation was symptomatic HF with New York Heart Association (NYHA) functional class $\geq$ III because of left ventricular dysfunction (end-diastolic diameter $\geq 55 \mathrm{~mm}$, ejection fraction (EF) $\leq 35 \%$ : confirmed by echocardiography using Simpson's method), despite optimized and stable medical therapy ( $>3$ months). All patients were implanted with a CRT device at the Division of Cardiology, University Hospital in Magdeburg after giving written and oral consent to the procedure and data collection. The research protocol was approved by the locally appointed ethics committee.

The etiology of HF was documented by coronary angiography in all patients before CRT implantation. Elderly patients were considered to be those aged 65 or older. Atrial fibrillation (AF) patients were considered to be only those presenting with permanent AF. The patients were subdivided into diabetic and non-diabetic groups according to the criteria of the Expert Committee on the Diagnosis and Classification of Diabetes! ${ }^{3}$

CRT was delivered using a left ventricular lead implanted in a lateral or posterolateral vein, in addition to the standard right ventricular and right atrial leads. In the patients in SR, the CRT devices were programmed into an atrial-synchronous biventricular pacing mode, with the lower rate limit set at 40 beats/min and upper rate limit at 130 beats/min. The atrioventricular delay was optimized by invasive monitoring of the pulse pressure or left ventricular first derivative pressure, as previously reported6 In patients with AF, the CRT devices were programmed in the VVIR mode with the lower rate limit set at 65 beats/min and the rate response individualized based on a short walking exercise.

None of the patients entered a training program, although spontaneous physical activity was encouraged. Patients were evaluated at the $1^{\text {st }} 3^{\text {rd }}$, and $6^{\text {th }}$ month after CRT implantation, and every 6 months thereafter. Echocardiographic evaluation and symptom-limited cardiopulmonary exercise testing were performed before and 6, 12, and 24 months after CRT implantation. Changes in medications or programming settings were recorded. Furthermore, at each follow-up visit, the stored data, including the arrhythmic episodes and device-based monitoring of the heart rate profile and heart rate variability, were retrieved and saved onto disks for further off-line analyses.

\section{Measurement of Daily Physical Activity}

All patients received CRT devices capable of continuous, automatic monitoring of physical activity (ie, Contak Renewal TR 2, Renewal I, II, IV; Guidant, St Paul, MN, USA) and which implemented the same algorithm, previously reported by Kadhiresan et al, for measuring the ALI! ${ }^{1}$ Briefly, ALI was defined as the percentage of time during which the acceleration exceeded a pre-set fixed threshold at $50 \mathrm{mG}\left(0.49 \mathrm{~m} / \mathrm{s}^{2}\right)$; this threshold corresponded to a treadmill walk speed of approximately $3.2 \mathrm{~km} / \mathrm{h}$, equivalent to 2.8 METS. The CRT devices automatically and continuously recorded the ALI, providing a daily mean value for the past week and a weekly mean value for the previous period. The mean value of the first week after implantation was considered to be the baseline. 
De-novo implanted patients- Raw Data

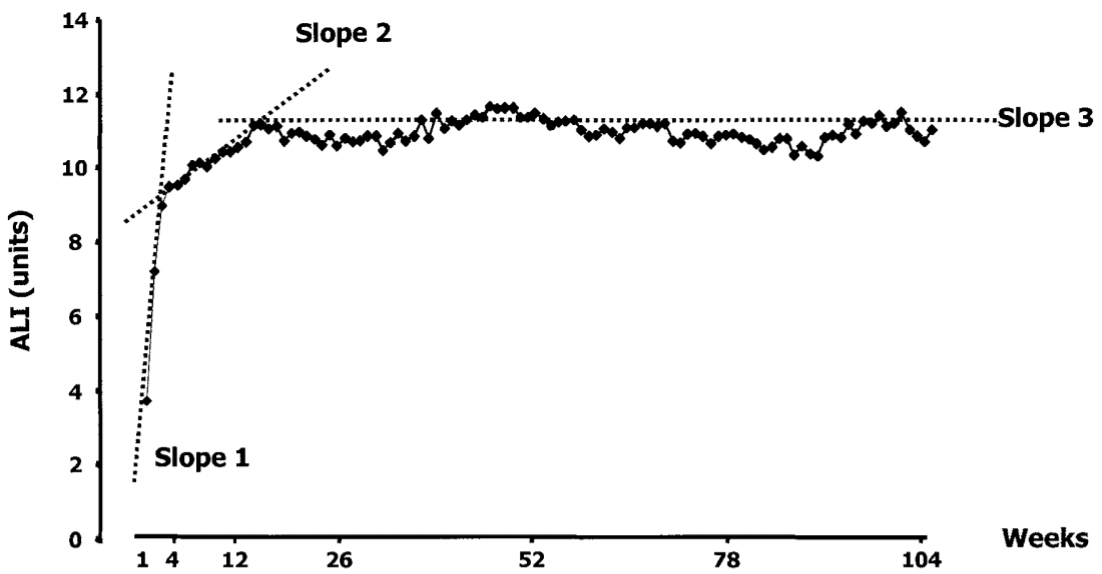

De-novo implanted patients- Fitted Data

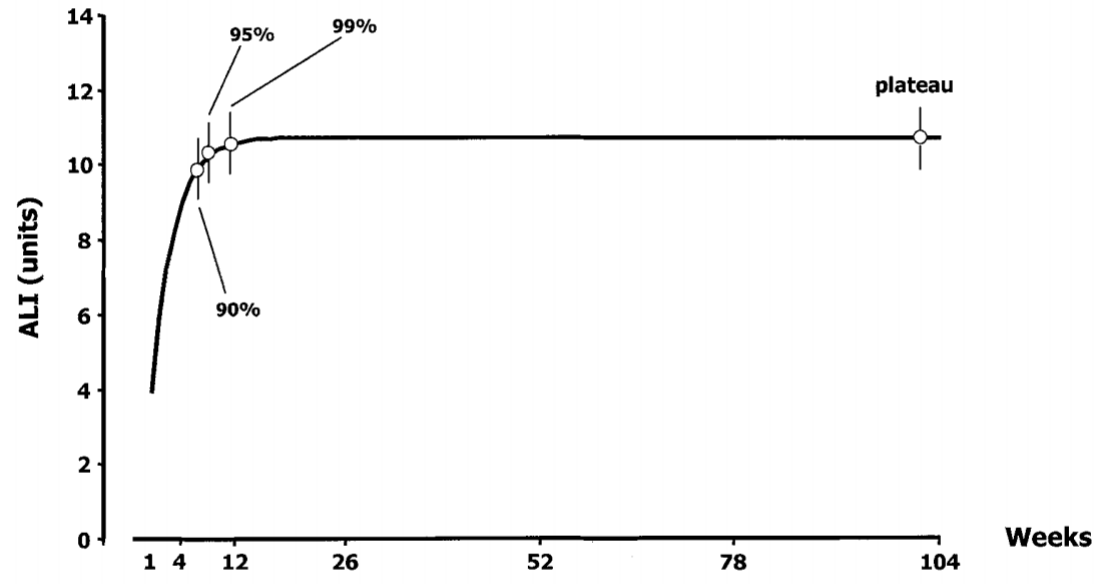

De-novo implanted and replaced patients

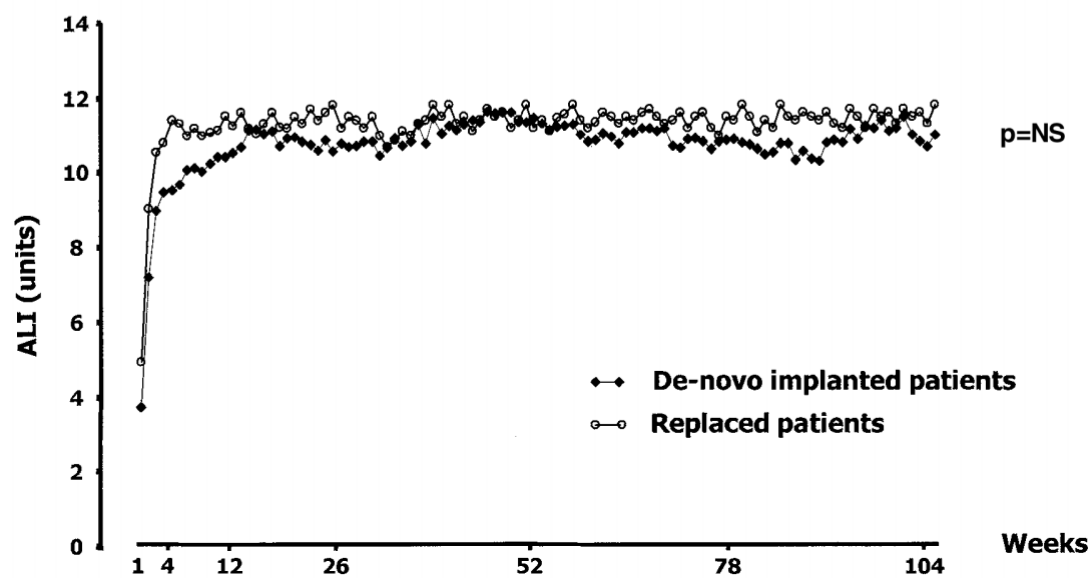

Fig 1. Effect of cardiac resynchronization therapy (CRT) on physical activity over the long-term follow-up: raw data (Upper panel) and data based on fitting (Middle panel) calculated according to the equation presented in Appendix 1, for the patients who received a new device; and raw data for de-novo implanted and device replacement patients (Bottom panel). The dotted lines in the upper panel represent the slopes of the 3 phases characterizing the changes in the Activity Log Index (ALI) after CRT implantation over the follow-up. The open circles in the middle panel show the mean and standard error corresponding to $90 \%, 95 \%$, and $99 \%$ of the plateau (based on fitting). The $\mathrm{p}$ value in the bottom panel refers to the comparison between the ALI values at the plateau in the de-novo implanted and replacement patients.

\section{Statistical Analysis}

All data are expressed as the mean \pm standard deviation. An exponential fitting equation (see Appendix 1) was used to describe the dynamic changes in the ALI over time, in particular to define the slope of the increase and the time to the plateau. The values at, and the time taken to reach, $90 \%$, $95 \%$, and $99 \%$ of the plateau have been defined. Paired and unpaired Student's t-test and the Chi-square test were used when appropriate. SPSS software version 10 was used (Chicago, IL, USA), and for all the tests the level of significance was set at $\mathrm{p}<0.05$.

\section{Results}

Table 1 presents the baseline demographic, clinical, and hemodynamic characteristics of all the patients enrolled in the study. Most of the patients were males $(80 \%)$ presenting with coronary artery disease $(60 \%)$ and stable SR $(81 \%)$. All the patients had a depressed left ventricular EF $(21 \pm 6 \%)$ and severely dilated left ventricles $(69 \pm 9 \mathrm{~mm})$. Approximately $41 \%$ of the patients had diabetes. Most of the patients (83\%) received a CRT device with a cardioverter-defibrillator back-up (CRT-D), which was indicated because of a 
Table 2 Long-Term Effect of CRT on Physical Activity, as Measured by the ALI, Oxygen Consumption at the Anaerobic Threshold and at Peak Exercise, in De-Novo Implanted HF Patients

\begin{tabular}{lrrrr}
\hline \hline & Baseline & 6 months & 12 months & 24 months \\
\hline Anaerobic threshold $\left(\mathrm{ml} \cdot \mathrm{kg}^{-1} \cdot \mathrm{min}^{-1}\right)$ & $9.5 \pm 2.2$ & $12.8 \pm 3.6$ & $13.0 \pm 1.7$ & $14.5 \pm 3.1$ \\
Peak exercise $\left(\mathrm{ml} \cdot \mathrm{kg}^{-1} \cdot \mathrm{min}^{-1}\right)$ & $13.4 \pm 4.2$ & $16.3 \pm 3.5$ & $16.5 \pm 2.8$ & $16.8 \pm 2.3$ \\
ALI (units) & $3.6 \pm 2.0$ & $10.7 \pm 4.4$ & $11.5 \pm 4.5$ & $11.5 \pm 5.0$ \\
\hline
\end{tabular}

ALI, Activity Log Index; HF, heart failure. Other abbreviation see in Table 1.

$\Delta$ of oxygen consumption at the anaerobic threshold vs $\Delta$ of the ALI: regression line $y=0.0133 x+83.857$, correlation coefficient $R=0.0073$.

$\Delta$ of oxygen consumption at the peak exercise vs $\Delta$ of the ALI: regression line $y=-0.5756 x+103.67$, correlation coefficient $R=$ -0.2696 .

history of ventricular arrhythmias $(21 \%)$ or for primary prevention $(79 \%)$. A few patients $(16 \%)$ received amiodarone for $\mathrm{AF}$ prevention or suppression of runs of ventricular tachycardia. Except for diuretics, which were frequently reduced, no further dosage adjustment of angiotensin-converting-enzyme inhibitors or angiotensin-receptor blockers was usually made. No patient was crossed over to any other $\beta$-blocking agent, but a dosage increase was attempted in 8 patients, 4-6 months after CRT implantation.

Most of the patients (83\%) were de-novo implanted, but in 31 patients the device was replaced $43 \pm 17$ months after the first implantation because of battery depletion. The denovo implanted patients differed from the others (Table 1 ) by presenting more often with an ischemic etiology (62 vs $43 \%, \mathrm{p}<0.05)$ and more frequently receiving a CRT-D device (90 vs $53 \%, \mathrm{p}<0.01$ ).

The older patients ( $\geq 65$ years, $n=68$ ) differed from the younger ones by presenting more frequently with an ischemic etiology (74 vs 53\%, p<0.03), diabetes (47 vs $22 \%$, $\mathrm{p}<0.01$ ), and less dilated left ventricle (end-diastolic diameter: $67 \pm 8$ vs $71 \pm 8 \mathrm{~mm}, \mathrm{p}<0.01$ ). Ischemic patients differed from the idiopathic ones in that they were more frequently male (88 vs $69 \%, \mathrm{p}<0.02)$ and presented with a significantly higher resting heart rate $(77 \pm 16$ vs $70 \pm 14$ beats/min, $\mathrm{p}<$ $0.03)$. The patients with permanent AF did not differ in any clinical, echocardiographic or hemodynamic parameters from those in SR. Similarly, the diabetic patients did not differ from the non-diabetic ones in any characteristic.

\section{Outcome After CRT}

Clinical follow-up and device interrogation were possible in all patients. At 1-year follow-up in the de-novo implanted

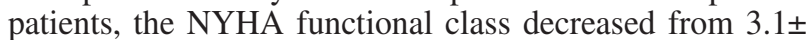
0.4 to $2.1 \pm 0.5(\mathrm{p}<0.01)$, left ventricular EF increased from $21 \pm 7$ to $23 \pm 8 \%(\mathrm{p}<0.01)$, left ventricular end-diastolic diameter decreased from $69 \pm 9$ to $66 \pm 8 \mathrm{~mm}(\mathrm{p}<0.01)$ and oxygen consumption at peak exercise improved from 13.4 \pm 4.2 to $16.5 \pm 2.8 \mathrm{ml} \cdot \mathrm{kg}^{-1} \cdot \mathrm{min}^{-1}(\mathrm{p}<0.01)$.

Over a mean follow-up period of $668 \pm 352$ days, 17 patients $(10 \%, 5$ of those with device replacement) died and 2 underwent urgent heart transplantation. The cause of death was HF in 7 patients, sudden cardiac death in 3 and non-cardiac causes in 7 . Twenty-five patients (14\%) received an appropriate shock from the CRT-D device because of ventricular fibrillation or fast ventricular tachycardia ( $>180$ beats/min). Seven patients (4\%) received an inappropriate shock from the CRT-D device because of the occurrence of rapidly conducting AF. Forty-three patients (24\%) were hospitalized at least once for cardiovascular reasons.

\section{Improvement of Physical Activity in De-Novo Implanted Patients}

Over the follow-up, in the de-novo implanted patients there was a large increase in the ALI shortly after device implantation. The ALI increased from a baseline value of $3.6 \pm 2.0$ to $11.2 \pm 4.6(\mathrm{p}<0.005)$ at 104 weeks after CRT initiation (Fig 1). The ALI changes were segregated into 3 phases: a first phase ( $>3$ weeks after implantation) showing a very steep slope (Fig 1); a second phase (from week 4 to week 12) characterized by a less steep increase in ALI, which was statistically different from that of the first phase $(p<0.05)$; finally, a third phase represented by a plateau (from week 13 up to week 104), the slope of which was again significantly different from that of the second phase $(\mathrm{p}<0.05)$ (Fig 1).

The ALI changes were best fitted by a monotonic exponential curve (Fig 1) and 90\%, 95\%, and 99\% of the plateau was reached $5.9 \pm 0.5,7.4 \pm 0.6$ and $11.8 \pm 1.0$ weeks, respectively, after CRT implantation.

Despite a significant increase in the ALI and oxygen consumption at the anaerobic threshold and at peak exercise, no statistically significant correlation was found between the changes in these parameters (Table 2).

In patients who experienced deterioration of $\mathrm{HF}$ (ie, death, heart transplantation, or hospitalization), the ALI showed various changes before the event. In some cases it decreased gradually from approximately 3-20 weeks before the episode; in others it decreased suddenly approximately 1-3 weeks before the event, whereas in some the ALI showed no obvious changes prior to the incident. The patients who died or underwent urgent heart transplantation did not show any difference in ALI as compared to the remainder of the population at any point in time during follow-up. There were no significant differences in the slopes in each phase between them, either.

\section{Replacement vs De-Novo Implanted Patients}

In patients in whom the CRT device was replaced, the

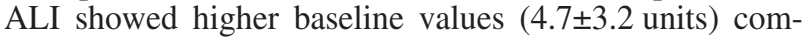
pared with those undergoing de-novo implantation (3.6 \pm 2.0 units, $\mathrm{p}<0.05)$, whereas plateau values did not differ. However, times to $90 \%, 95 \%$, and $99 \%$ of plateau were significantly $(\mathrm{p}<0.001)$ longer in the de-novo implanted patients than in the patients undergoing replacement. Consistently, the slopes of phases 1 and 3 were similar in these 2 subgroups ( $\mathrm{p}=\mathrm{NS}$ for both slopes), whereas the slope of phase 2 was significantly steeper in the de-novo implanted patients $(\mathrm{p}<0.05)$, being flat in the replacement group. Fig 1 shows that, compared with the replacement patients who had almost reached the plateau at 4 weeks after re-implantation, the de-novo implanted patients had a continuous increase in the ALI in the period between weeks 4 and 12 . 

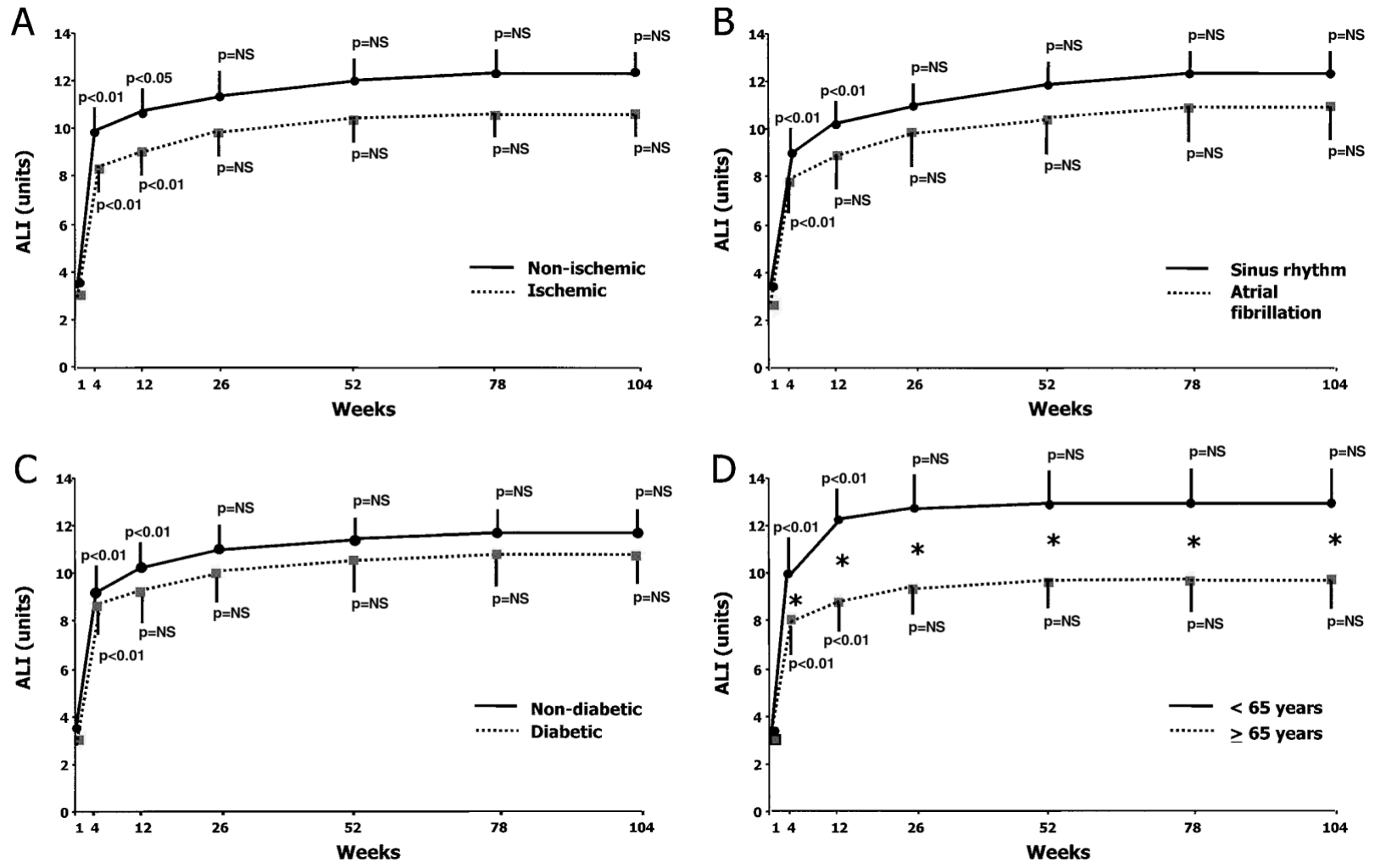

Fig 2. Effect of the etiology of heart failure (A), underlying rhythm (B), diabetes (C), and age (D) on the improvement in device-based measurement of physical activity in patients implanted with a new cardiac resynchronization therapy device. The vertical bars represent the standard errors. The $p$ values refer to the comparison between each follow-up and the previous one in each subgroup. *Statistically significant difference between the subgroups at each follow-up. ALI, Activity Log Index.

Influence of Etiology, Heart Rhythm, Diabetes, and Age on ALI

The analysis of ALI improvement after CRT in different HF populations was performed only among the de-novo implanted patients. No statistically significant difference in the extent of physical activity at baseline was found between the ischemic and non-ischemic patients, between patients in SR and those with permanent AF, or between diabetic and non-diabetic patients (Fig 2). Similarly, the ALI values reached at any point in time, and in particular at the end of the first and second phases and the plateau, did not significantly differ between the subgroups.

Although the younger ( $<65$ years old) and older $(\geq 65$ years old) patients had similar ALIs at baseline, the younger patients reached significantly higher values during the entire follow-up and at the plateau $(13.3 \pm 4.8$ vs $9.5 \pm$ 4.2, $\mathrm{p}<0.001)($ Fig 2).

\section{Discussion}

This study shows that long-term device-based monitoring of the physical activity of HF patients treated with CRT is feasible. CRT resulted in a large and long-term sustained improvement in spontaneous physical activity, as arbitrarily measured by the ALI. This improvement was accompanied by, but not statistically correlated with, increases in oxygen consumption both at the anaerobic threshold and at peak exercise. The patients with different etiologies and rhythms, as well as diabetic and non-diabetic patients, obtained similarly large and sustained functional benefits from CRT. In contrast, there was an age-related difference in the magnitude of the increase in physical activity after CRT.

\section{Sustained Benefit of CRT on Physical Activity}

Braunschweig et al recently reported that in a limited number of patients followed up for 12 weeks automatic device-based monitoring of the physical activity was feasible, ${ }^{12}$ and our data have confirmed that result in a much larger population with a longer follow-up and using different technology.

As shown in Fig 1, the low ALI during the first week after initiation of CRT was clearly related to post-implantation recovery. The steep increase in the ALI during the 3 weeks following implantation was probably still related to recovery after hospitalization. That hypothesis was supported by the overlapping of the phase between the de-novo and re-implanted patients. The second phase, from week 4 to 12 , during which a continuous increase in ALI was observed, may represent the improvement in physical activity induced by CRT. Indeed, this phase was noted exclusively in the patients who received a de-novo device, whereas the patients with device replacement had already almost reached the plateau by week 4 after re-implantation. Further, the plateau values were similar for both subgroups and phase 3 expressed the long-term, sustained effects of CRT on physical activity.

The changes in physical activity resembled the effect of a training program, 3 but occurred without formal participation in such a program. The increased functional capacity over the follow-up was probably because of improved car- 
diac performance ${ }^{14,15}$ and stroke volume, 5 reduced mitral regurgitation, ${ }^{16,17}$ and reduced sympathetic drive to the heart5, 8 Moreover, the patients' motivation to do physical activity may have increased as exercise tolerance improved, by interrupting the vicious cycle of exertional dyspnea leading to less willingness to exercise, reduction in the endurance capacity, increased sympathetic drive, higher resting heart rate causing exertional dyspnea, and so forth.

\section{Improvement in the Physical Activity of Different HF Population Groups During CRT}

For the first time, we have reported the effect of CRT on the spontaneous physical activity of different HF population groups. Whether CRT is equally beneficial in ischemic and non-ischemic patients, and in those in SR or with AF, is still a matter of debate. Our data demonstrated a similar improvement in the spontaneous physical activity induced by CRT in ischemic and non-ischemic patients, as well as in those with SR or AF. These findings confirm the recent reports on outcome after CRT in HF patients with different etiologies or underlying rhythms ${ }^{18,19}$

Diabetes is an important comorbidity in HF patients, significantly worsening their prognosis. ${ }^{20}$ Usually, diabetic patients have a lower functional capacity, despite the best optimized therapy for $\mathrm{HF}^{21}$ Our data showed that CRT induced a similar improvement in physical activity in diabetic and non-diabetic patients. Increased exercise tolerance in diabetic patients treated with CRT may lead to an improved prognosis, but this needs to be tested in a larger prospective randomized study.

We have also showed that CRT improved the physical activity of HF patients older than 65 years of age. We arbitrarily set the cut-off for age, based on the mean value of our study cohort. Patients older than 65 years improved less than the younger patients, which is not surprising considering that degenerative processes, independent of HF, reduce the endurance capability of older patients. Nevertheless, in both age groups the increase in the physical activity was large and sustained over the long-term following the initiation of CRT. An increased level of spontaneous physical activity, especially in older patients, may greatly contribute to an improved feeling of well-being. Thus, based on the present data, CRT may be prescribed in older HF population cohorts; however, the effect of CRT in the geriatric population ( $>75$ years old) needs to be further investigated as only $7 \%$ of the present population was represented by patients over 75 years of age at the time of CRT implantation.

\section{Issues Related to ALI Monitoring}

Although ALI monitoring can be a useful objective tool for identifying patients who continue to have limited physical activity even after CRT, we were not able to determine a cut-off for the ALI changes that are predictive of different outcomes. There were no significant differences in the ALI values or the slopes in each phase between the patients who died or underwent urgent heart transplantation and the remainder of the population during follow-up. Moreover, it might be difficult to detect a deterioration of HF in the early phase only by monitoring the ALI values because they showed a variety of changes among the patients before worsening of HF. In contrast, the distance walked during $6 \mathrm{~min}$ has been proven to be predictive of outcome! This finding may question the value of the ALI as a surrogate endpoint for prognosis. A large inter-individual variability in ALI or the method of calculating ALI could be the source of such a difference. Physical training improves outcome $^{3}$ as results of increasing endurance capacity and the magnitude of the physical activity. With the devices used in the present study, the ALI represented the percentage of time during which acceleration exceeded a pre-set fixed threshold at $50 \mathrm{mG}$ corresponding to $3.2 \mathrm{~km} / \mathrm{h}$. Thus, it is possible that because of improved hemodynamic status, many patients experienced prolonged physical activity throughout the day but which was not strenuous enough to overcome the pre-defined acceleration threshold. The value may have been too high to evaluate the improvement in physical activity in elderly and untrained patients. Thus, an individualized threshold for ALI measurements may be more desirable.

The considerable increase in the ALI values corresponded to improvement in oxygen consumption at both the peak exercise and the anaerobic threshold, although there was no statistically significant correlation between the changes in these parameters, in contrast to a previous report.11 The lack of strong associations between them in the present study is likely explained, in part, by the fact that they assess different aspects of functional status, reflecting different facets of health status, and perhaps the more indicative way of assessing a patient's true functional impairment is to measure activity during normal daily life, which formal laboratorybased exercise tests do not truly represent. As laboratorybased exercise testing is a somewhat 'unnatural' form of exercise that bears little relation to typical activity patterns, their usefulness as a true representation of patients' abilities is questionable. Scores of daily activity may be influenced much more by psychological or other factors, in addition to physical capability.

Daily activity levels measured with pedometers bear no relation to patients' performance in laboratory-based exercise tests ${ }^{22}$ however, it has been demonstrated that the pedometer score is a powerful predictor of survival in chronic HF and appears to be more powerful than laboratory-based exercise tests. 23 Therefore, this type of less artificial means of continuous daily assessment is valuable in identifying patients at high risk and provides an objective measure of incapacity over a longer time period within the normal everyday environment. The ALI value, a novel monitoring feature that is an objective measurement of functional capacity, has potential for wide clinical use, and the impact of this on HF management should be further evaluated in larger controlled studies.

\section{Study Limitations}

One limitation of our study was the lack of ALI evaluation before CRT implantation or during the no treatment period (CRT-off period), which could validate our conclusions. We tried to overcome this limitation by comparing the time-course of the ALI values observed between the denovo implanted and re-implanted patients. In that way we were able to appreciate the influence of the recovery phase and to limit it to the first 3 weeks after implantation. This hypothesis is supported by the overlapping of this phase in the de-novo and re-implanted patients. Furthermore, the second phase (from weeks 4 to 12 ), which very likely reflected the real improvement in physical activity induced by CRT, was noted exclusively in patients who received a de-novo device. Whether CRT will improve the physical activity of HF patients needs to be further investigated.

Another possible limitation of our study was the lack of 
a control group that underwent a coached training program. A technical limitation of ALI may be that it represents the weekly mean value of physical activity, which may have obscured clinically meaningful changes in physical activity during the week. Furthermore, we did not evaluate the quality of life of the present patients.

\section{Conclusions}

Long-term, device-based automatic measurement of physical activity, as assessed by ALI, in HF patients treated with CRT is feasible. CRT significantly improved long-term physical activity in HF patients with a ventricular conduction delay. The improvement in physical activity was sustained, resembling the effect of a training program. The magnitude of the ALI increases was similar, regardless of HF etiology, underlying rhythm, or the presence of diabetes. Older patients had a lower, yet stable, increase in ALI as compared with younger patients.

\section{References}

1. Bittner V, Weiner DH, Yusuf S, Rogers WJ, McIntyeare KM, Bangdiwala SI, et al. Prediction of mortality and morbidity with a 6-minute walk test in patients with left ventricular dysfunction: SOLVD Investigators. JAMA 1993; 270: 1702-1707.

2. Barengo NC, Hu G, Lakka TA, Pekkarinen $\mathrm{H}$, Nissinen A, Tuomilehto J. Low physical activity as a predictor for total and cardiovascular disease mortality in middle-aged men and women in Finland. Eur Heart J 2004; 25: 2204-2211.

3. Belardinelli R, Georgiou D, Cianci G, Purcaro A. Randomized, controlled trial of long-term moderate exercise training in chronic heart failure: Effects on functional capacity, quality of life, and clinical outcome. Circulation 1999; 99: 1173-1182.

4. Brodie DA, Inoue A. Motivational interviewing to promote physical activity for people with chronic heart failure. J Adv Nurs 2005; 50: $518-527$.

5. Abraham WT, Fisher WG, Smith AL, Delurgio DB, Leon AR, Loh E, et al; MIRACLE Study Group. Multicenter InSync Randomized Clinical Evaluation. Cardiac resynchronisation in chronic heart failure. N Engl J Med 2002; 346: 1845-1853.

6. Auricchio A, Stellbrink C, Sack S, Block M, Vogt J, Bakker P, et al; Pacing Therapies in Congestive Heart Failure (PATH-CHF) Study Group. Long-term clinical effect of hemodynamically optimised cardiac resynchronisation therapy in patients with heart failure and ventricular conduction delay. J Am Coll Cardiol 2002; 39: 2026-2033.

7. Auricchio A, Kloss M, Trautmann SI, Rodner S, Klein H. Exercise performance following cardiac resynchronization therapy in patients with heart failure and ventricular conduction delay. Am J Cardiol 2002; 89: 198-203.

8. Adamson PB, Smith AL, Abraham WT, Kleckner KJ, Stadler RW, Shih A, et al; InSync III Model 8042 and Attain OTW Lead Model 4193 Clinical Trial Investigators. Continuous autonomic assessment in patients with symptomatic heart failure: Prognostic value of heart rate variability measured by an implanted cardiac resynchronization device. Circulation 2004; 110: 2389-2394.

9. Cazeau S, Leclercq C, Lavergne T, Walker S, Varma C, Linde C, et al. Effects of multisite biventricular pacing in patients with heart failure and intraventricular conduction delay. $N$ Engl J Med 2001; 3: $873-880$.

10. Domenico Pinna G, Opasich C, Mazza A, Tangenti A, Maestri R, Sanarico M. Reproducibility of the six-minute walking test in chronic heart failure patients. Stat Med 2000; 19: 3087-3094.

11. Kadhiresan VA, Pastore J, Auricchio A, Sack S, Doelger A, Girouard $\mathrm{S}$, et al; PATH-CHF Study Group. Pacing therapies in congestive heart failure: A novel method - the activity log index - for monitoring physical activity of patients with heart failure. Am J Cardiol 2002; 89: $1435-1437$.

12. Braunschweig F, Mortensen P, Gras D, Reiser W, Lawo T, Mansour $\mathrm{H}$, et al. Monitoring of physical activity and heart rate variability in patients with chronic heart failure using cardiac resynchronisation therapy. Am J Cardiol 2005; 95: 1104-1107.

13. The Expert Committee on the Diagnosis and Classification of Diabetes Mellitus. Report of the Expert committee on the Diagnosis and Classification of Diabetes Mellitus. Diabetes Care 1998; 21: S5-S19.
14. Kass DA, Chen CH, Curry C, Talbot M, Berger R, Fetics B, et al. Improved left ventricular mechanics from acute VDD pacing in patients with dilated cardiomyopathy and ventricular conduction delay. Circulation 1999; 99: 1567-1573.

15. Auricchio A, Stellbrink C, Block M, Sack S, Vogt J, Bakker P, et al. Effect of pacing chamber and atrioventricular delay on acute systolic function of paced patients with congestive heart failure: The Pacing Therapies for Congestive Heart Failure Study Group. Circulation 1999; 99: 2993-3001.

16. Breithardt OA, Sinha AM, Schwammenthal E, Bidaoui N, Markus $\mathrm{KU}$, Franke A, et al. Acute effects of cardiac resynchronization therapy on functional mitral regurgitation in advanced systolic heart failure. J Am Coll Cardiol 2003; 41: 765-770.

17. Abraham WT, Young JB, Leon AR, Adler S, Bank AJ, Hall SA, et al; Multicenter InSync ICD II Study Group. Effects of cardiac resynchronization on disease progression in patients with left ventricular systolic dysfunction, an indication for an implantable cardioverter-defibrillator, and mildly symptomatic chronic heart failure. Circulation 2004; 110: $2864-2868$.

18. Molhoek SG, Bax JJ, van Erven L, Bootsma M, Boersma E, Steendijk $\mathrm{P}$, et al. Comparison of benefits from cardiac resynchronization therapy in patients with ischemic cardiomyopathy versus idiopathic dilated cardiomyopathy. Am J Cardiol 2004; 93: 860-863.

19. Molhoek SG, Bax JJ, Bleeker GB, Boersma E, van Erven L, Steendijk $\mathrm{P}$, et al. Comparison of response to cardiac resynchronization therapy in patients with sinus rhythm versus chronic atrial fibrillation. Am J Cardiol 2004; 94: 1506-1509.

20. Tenenbaum A, Motro M, Fisman EZ, Leor J, Freimark D, Boyko V, et al. Functional class in patients with heart failure is associated with the development of diabetes. Am J Med 2003; 114: 271-275.

21. Suskin N, McKelvie RS, Burns RJ, Latini R, Pericak D, Probstfield J, et al. Glucose and insulin abnormalities relate to functional capacity in patients with congestive heart failure. Eur Heart J 2000; 21: $1368-1375$.

22. Houghton AR, Harrison M, Cowley AJ, Hampton JR. Assessing exercise capacity, quality of life and haemodynamics in heart failure: Do the tests tell us the same thing? Eur J Heart Fail 2002; 4: 289-295.

23. Walsh JT, Charlesworth A, Andrews R, Hawkins M, Cowley AJ. Relation of daily activity levels in patients with chronic heart failure to long-term prognosis. Am J Cardiol 1997; 79: 1364-1369.

24. Nocel J, Wright SJ. Numerical optimization. New York, NY, USA: Springer; 1999; $251-266$.

25. See http://www.netlib.org/slatec/ Provider: The U.S. Department of Energy Oak Ridge National Laboratory. Date: December 2004.

26. Kirkup L. Principles and applications of non-linear least squares: An Introduction for physical scientists using Excel's Solver. October 2003. Available from the Department of Applied Physics, University of Technology Sydney at http://www.science.uts.edu.su/physics/ nonlin $2003 . \mathrm{html}$

27. Heck A. Introduction to Maple, 3rd edn. New York, NY, USA: Springer-Verlag; 2003

28. Taylor JR. An introduction to error analysis. Sausalito, California, USA: University Science Books; 1997.

\section{Appendix 1}

This appendix describes the technique used to compute the exponential fit to the Activity Log Index (ALI) data. Assuming we had $M$ patients, for each patient $i=1, \cdots, M$ we had $m_{i}$ data points $\left(t_{i, j}, y_{i, j}\right)$ with $j=1, \cdots, m_{i}$. The first subscript $i$ refers to the patient number and the second subscript $j$ refers to an individual data point for that patient consisting of the week after the implant $t_{i, j}$ (the implantation week is by definition week 1) and the value of the ALI at that week $y_{i, j}$. We were interested in fitting a single exponential curve to all of the data, (ie, to all $\left(t_{i, j}, y_{i, j}\right)$ for all $j$ and all $i$ ). We let $\left(T_{n}, Y_{n}\right)$ be the data points drawn from the aggregated set of data for all the patients,

$$
\left(T_{n}, Y_{n}\right) \in \underset{\substack{j=1, \ldots, m_{i} \\ i=1, \cdots, M}}{\mathrm{U}}\left(t_{i, j}, y_{i, j}\right)
$$

where $n=1, \cdots, N$ and $N=\sum_{i=1}^{\mathrm{M}} m_{i}$. We fit this aggregated data into the exponential

$$
\hat{Y}(T)=a+b\left(1-e^{-\frac{T-1}{c}}\right) \text {. }
$$

The regression coefficients $a, b$, and $c$ in [2] were computed by the method of non-linear least squares. This technique minimized the sum of the squares of the residuals 


$$
\frac{1}{2} \sum_{n=1}^{N}\left(\hat{Y}\left(T_{n}\right)-Y_{n}\right)^{2} .
$$

We employed a modified Levenberg-Maquardt ${ }^{24}$ method to perform this minimization and computed the regression coefficients. This was performed using a custom Fortran 95 computer program that incorporated the DNLS1 routine from the SLATEC Common Mathematical Library25

The standard errors for the regression coefficients were given by Kirkup ${ }^{26}$

$$
\sigma_{a}=\sigma \sqrt{E_{1,1}^{-1}},
$$

$\sigma_{b}=\sigma \sqrt{E_{2,2}^{-1}}$, and

$$
\sigma_{c}=\sigma \sqrt{E_{3,3}^{-1}}
$$

where $\sigma$ was ginen by

$$
\sigma \approx \sqrt{\frac{\sum_{n=1}^{N}\left(\hat{Y}\left(T_{n}\right)-Y_{n}\right)^{2}}{N-3}} .
$$

The $E_{1,1}^{-1}, E_{2,2}^{-1}$, and $E_{3,3}^{-1}$ were the diagonal elements of the inverse of the matrix of partial derivatives

$$
\begin{aligned}
& E=\left(\begin{array}{ccc}
\sum_{n-1}^{N}\left[\frac{\partial \hat{Y}\left(T_{n}\right)}{\partial a}\right]^{2} & \sum_{n-1}^{N}\left[\frac{\partial \hat{Y}\left(T_{n}\right)}{\partial a} \frac{\partial \hat{Y}\left(T_{n}\right)}{\partial b}\right] \sum_{n-1}^{N}\left(\frac{\partial \hat{Y}\left(T_{n}\right)}{\partial a} \frac{\partial \hat{Y}\left(T_{n}\right)}{\partial c}\right] \\
\sum_{n-1}^{N}\left[\frac{\partial \hat{Y}\left(T_{n}\right)}{\partial a} \frac{\partial \hat{Y}\left(T_{n}\right)}{\partial b}\right] & \sum_{n-1}^{N}\left[\frac{\partial \hat{Y}\left(T_{n}\right)}{\partial b}\right]^{2} & \sum_{n-1}^{N}\left(\frac{\partial \hat{Y}\left(T_{n}\right)}{\partial b} \frac{\partial \hat{Y}\left(T_{n}\right)}{\partial c}\right] \\
\sum_{n-1}^{N}\left[\frac{\partial \hat{Y}\left(T_{n}\right)}{\partial a} \frac{\partial \hat{Y}\left(T_{n}\right)}{\partial c}\right] \sum_{n=1}^{N}\left[\frac{\partial \hat{Y}\left(T_{n}\right)}{\partial b} \frac{\partial \hat{Y}\left(T_{n}\right)}{\partial c}\right] & \sum_{n-1}^{N}\left[\frac{\partial \hat{Y}\left(T_{n}\right)}{\partial c}\right]^{2}
\end{array}\right] \\
& E=\left(\begin{array}{ccc}
\sum_{n-1}^{N}\left[\frac{\partial \hat{Y}\left(T_{n}\right)}{\partial a}\right]^{2} & \sum_{n-1}^{N}\left[\frac{\partial \hat{Y}\left(T_{n}\right)}{\partial a} \frac{\partial \hat{Y}\left(T_{n}\right)}{\partial b}\right] \sum_{n-1}^{N}\left(\frac{\partial \hat{Y}\left(T_{n}\right)}{\partial a} \frac{\partial \hat{Y}\left(T_{n}\right)}{\partial c}\right] \\
\sum_{n-1}^{N}\left[\frac{\partial \hat{Y}\left(T_{n}\right)}{\partial a} \frac{\partial \hat{Y}\left(T_{n}\right)}{\partial b}\right] & \sum_{n-1}^{N}\left[\frac{\partial \hat{Y}\left(T_{n}\right)}{\partial b}\right]^{2} & \sum_{n-1}^{N}\left(\frac{\partial \hat{Y}\left(T_{n}\right)}{\partial b} \frac{\partial \hat{Y}\left(T_{n}\right)}{\partial c}\right] \\
\sum_{n-1}^{N}\left[\frac{\partial \hat{Y}\left(T_{n}\right)}{\partial a} \frac{\partial \hat{Y}\left(T_{n}\right)}{\partial c}\right] \sum_{n=1}^{N}\left[\frac{\partial \hat{Y}\left(T_{n}\right)}{\partial b} \frac{\partial \hat{Y}\left(T_{n}\right)}{\partial c}\right] & \sum_{n-1}^{N}\left[\frac{\partial \hat{Y}\left(T_{n}\right)}{\partial c}\right]^{2}
\end{array}\right] \\
& E=\left(\begin{array}{ccc}
\sum_{n-1}^{N}\left[\frac{\partial \hat{Y}\left(T_{n}\right)}{\partial a}\right]^{2} & \sum_{n-1}^{N}\left[\frac{\partial \hat{Y}\left(T_{n}\right)}{\partial a} \frac{\partial \hat{Y}\left(T_{n}\right)}{\partial b}\right] \sum_{n-1}^{N}\left(\frac{\partial \hat{Y}\left(T_{n}\right)}{\partial a} \frac{\partial \hat{Y}\left(T_{n}\right)}{\partial c}\right] \\
\sum_{n-1}^{N}\left[\frac{\partial \hat{Y}\left(T_{n}\right)}{\partial a} \frac{\partial \hat{Y}\left(T_{n}\right)}{\partial b}\right] & \sum_{n-1}^{N}\left[\frac{\partial \hat{Y}\left(T_{n}\right)}{\partial b}\right]^{2} & \sum_{n-1}^{N}\left(\frac{\partial \hat{Y}\left(T_{n}\right)}{\partial b} \frac{\partial \hat{Y}\left(T_{n}\right)}{\partial c}\right] \\
\sum_{n-1}^{N}\left[\frac{\partial \hat{Y}\left(T_{n}\right)}{\partial a} \frac{\partial \hat{Y}\left(T_{n}\right)}{\partial c}\right] \sum_{n=1}^{N}\left[\frac{\partial \hat{Y}\left(T_{n}\right)}{\partial b} \frac{\partial \hat{Y}\left(T_{n}\right)}{\partial c}\right] & \sum_{n-1}^{N}\left[\frac{\partial \hat{Y}\left(T_{n}\right)}{\partial c}\right]^{2}
\end{array}\right]
\end{aligned}
$$

The inverse was computed analytically and automatically converted to Fortran 95 for inclusion in the computer code using a Maple ${ }^{\mathrm{TM}}$ Computer Algebra System? 27

The exponential in [2] asymptotes to the value $a+b$ as $\mathrm{T} \rightarrow \infty$. For finite times, we defined the effective time at the data plateaus as the time at which [2] was within $90 \%, 95 \%$, or $99 \%$ of the asymptotic value $a+b$. This time was given by

$$
T_{p}=1-c \ln (1-\alpha)
$$

wherea was given by $0.9,0.95$, or 0.99 for the time at which the data was within $90 \%, 95 \%$, or $99 \%$ of the asymptotic plateau, respectively. Using conventional error propagation analysis ${ }^{28}$ the standard error for the plateau times was

$\sigma_{t p} \leq \sigma_{c} \ln (1-\alpha)$. 